\title{
El Consejo Aeronáutico Nacional: Análisis de su situación actual en el marco institucional de la Aeronáutica Civil Hondureña
}

\author{
Ana Lucía Ulloa Cadalso \\ Alexander J. Flores Moncada
}

\section{Resumen}

En Honduras la aeronáutica civil se rige por la Ley de Aeronáutica Civil de 2004 y en esta se establece el Consejo Aeronáutico Nacional (CAN) como un órgano consultivo que reúne a varias organizaciones estatales y de la sociedad hondureña con el objetivo principal de identificar, concertar y proponer recomendaciones que apoyen y contribuyan al logro de los objetivos de la ley referida. Considerando que la aeronáutica civil se relaciona con diversos temas nacionales tales como seguridad, turismo, medio ambiente, economía, entre otras, es importante que los diferentes sectores se coordinen y tomen decisiones consensuadas que permitan un desarrollo sostenido, ordenado y consistente de la aeronáutica civil. Dado lo anterior se describe la situación del CAN, la relación de sus integrantes con este y sus propuestas, se da a conocer cuál ha sido la experiencia que otros países como Chile, Ecuador y República Dominicana han tenido con este tipo de órganos y cuáles son las características que les han permitido tener éxito en sus esfuerzos de desarrollar su aeronáutica civil, todo lo anterior con el objetivo de aprender, adaptar, mejorar y adoptar buenas prácticas que complementen los esfuerzos nacionales para lograr un órgano colegiado fortalecido capaz de potenciar el desarrollo de la aeronáutica civil hondureña. Las recomendaciones propuestas giran en torno a la necesidad de hacer funcionar el CAN. Sin embargo, el simple funcionamiento no basta, es necesario que esta entidad adquiera un estatus de órgano ejecutivo facultado para plantear, definir, consensuar y llevar la práctica una política de Estado para el desarrollo de la aeronáutica civil en Honduras, este nivel de deliberación ha sido, a juicio de los autores de la investigación, la clave del éxito en los países en donde los homólogos del CAN han funcionado haciendo aportes relevantes.

Palabras clave: Aeronáutica, Consejo Aeronáutico Nacional, aeronáutica civil hondureña, sistema aeroportuario 


\section{Abstract:}

In Honduras the civil aeronautics is directed by the Civil Aeronautic Law of 2004, in this law is established the National Aeronautic Council (CAN in Spanish) like an advisory body that includes several state entities and Honduran society with the principal objective of identify, arrange and propose recommendations that support and contribute to reach the objectives of the referred law. Considering that the civil aeronautics is related with many national issues like security, tourism, environment, economy and others, is important that the different sectors be coordinated and take decisions by consensus, all this will allow a sustainable, ordered and consistent development of civil aeronautics, all this entities will be together in the NAC. It has been described the situation of the NAC, relations between members and their proposals; also this research gives a general view of the experience that other countries like Chile, Ecuador and Dominican Republic that already have similar council organs like the NAC experienced, and mentioned what could be some of the strategies and strengthens that allowed them to success in the develop the civil aeronautics in their countries; all this with the objective of learn, adapt, improve y adopt good practices that turns around the need of operate the NAC in Honduras. However, just the operation is not enough, is necessary that this entity gets an executive organ status that could have the faculty of planning, define, agree and put in practice a State policy for the development of the civil aeronautics in Honduras, this level of deliberation has been, as the authors of this research believe, the clue of the success in the countries where the NAC's similar organs has worked giving relevant contributions.

Keywords: Aeronáutics, Aeronáutical National Council, Honduran Civil Aeronautics, Airport System.

Ana Lucía Ulloa Cadalso, (ana.ulloa@unah.edu.hn), Departamento de Ciencias Aeronáuticas Facultad de Ciencias Espaciales, Universidad Nacional Autónoma de Honduras - UNAH. Alexander J. Flores Moncada, (alexander.flores@unah.edu.hn), Diplomado en Gestión de Sistemas Aeroportuarios Universidad Nacional Autónoma de Honduras UNAH 


\section{Introducción}

Hoy en día el tema de la aeronáutica civil en Honduras ha tomado mayor relevancia dentro de la agenda nacional pues tanto en el ámbito académico como gubernamental se visualizan esfuerzos concretos para potenciar su desarrollo. Existen dos hechos que fundamentan lo antes descrito: el primero es la iniciativa del gobierno central de desarrollar proyectos de infraestructura aeroportuaria en diferentes departamentos del Estado hondureño como Comayagua, Lempira y Copán, entre otros; y el segundo es la creación del Departamento de Ciencias Aeronáuticas dentro de la Facultad de Ciencias Espaciales de la Universidad Nacional Autónoma de Honduras (UNAH).

Como institución académica, el Departamento de Ciencias Aeronáuticas tiene tres funciones adicionales a la de proveer al país profesionales altamente calificados; nos referimos al de la docencia, la vinculación con la sociedad y la investigación, funciones que cumple, en parte, al generar nuevos programas de estudio, diplomados, seminarios y carreras universitarias relacionadas con la industria aeronáutica.

Con lo antes descrito, no se puede ignorar el importante rol de la Universidad Nacional Autónoma de Honduras a través de sus unidades académicas como el Depto. de Ciencias Aeronáuticas en el desarrollo integral del país, puesto que es a través de esta institución que se estudian científicamente situaciones de carácter nacional, que la sociedad en general y que el gobierno desconocen o ignoran, que conllevan al planteamiento de recomendaciones para el mejoramiento de las mismas mediante la innovación.

Es por esto que de una actividad académica de la UNAH, el primer Diplomado en Gestión de Sistemas Aeroportuarios impartido en el país, nace la discusión de temas relevantes propios de la situación aeronáutica civil del país, destacándose el tema del Consejo Aeronáutico Nacional (CAN), y es que de éste sólo se conocía qué era y quienes lo integraban pero no así su situación actual en el marco institucional de la aeronáutica civil del país. 
La falta de implementación del CAN es evidente cuando al buscar información oficial sobre éste, la única referencia es la Ley de Aeronáutica Civil de Honduras. De igual manera, es evidente cuando desde el 2004, periodo en el que se aprueba y publica la Ley de Aeronáutica Civil de Honduras, al tomar decisiones relacionadas al ámbito aeronáutico civil, los diferentes entes estatales lo hacen sin la opinión de sectores de la sociedad hondureña que pueden ser afectados. Un ejemplo importante de lo anterior fue el cierre parcial y temporal del Aeropuerto Internacional de Toncontín por parte del Poder Ejecutivo, a raíz del accidente aéreo de mayo de 2008, sin que previamente, con la implementación del CAN, se consultara a los sectores económicos, quienes se vieron afectados por la paralización de las actividades económicas propias del ámbito aeronáutico.

\section{Marco Teórico}

La Dirección General de Aeronáutica Civil, actualmente la Agencia Hondureña de Aeronáutica, aporta la visión técnica propia de la aeronáutica civil pero no de la económica, social y ambiental; Es así que temas como el narcotráfico, medidas para la protección y conservación del medio ambiente, la construcción y habilitación de nuevos aeropuertos en las diversas regiones del país y sucesos propios de la aeronáutica civil (des categorización de país) que requieran de una gestión como Estado y la participación de la sociedad civil deben ser tratados desde una organización u órgano que aglutine los representantes de los diferentes sectores nacionales desde el estatal al económico y social. Como se ha afirmado anteriormente, el CAN no ha sido ese órgano que facilite la interacción de estos representantes para el tratamiento de los temas ya mencionados pues cada vez que se toman decisiones desde la institucionalidad del Estado, los diferentes sectores de la sociedad manifiestan diferentes opiniones y en ciertas situaciones no emiten opinión alguna y es precisamente lo anterior lo que puede estar afectando el desarrollo de la aeronáutica civil nacional.

El Estado de Honduras no debe perder más tiempo en relación al desarrollo del ámbito aeronáutico civil pues los retos del siglo XXI a nivel nacional e internacional como los temas de seguridad, medio ambiente y crisis económica deben ser enfrentados y superados de manera organizada y el pleno funcionamiento del CAN podría ser la clave para lograrlo ya que éste sería el espacio donde convergerían los diferentes sectores involucrados en el ámbito aeronáutico como también de aquellos que se ven afectados por la industria y por tanto contribuyendo con diferentes puntos de vista en la discusión sobre temas aeronáuticos nacionales. 
La Ley de Visión de País (2010-2038) y Plan de Nación (2010-2022) de la República de Honduras expresa: "La infraestructura productiva (energía, transporte y comunicaciones) es un medio importante para propiciar el desarrollo social de los pueblos y el desencadenamiento del potencial productivo de las naciones, Honduras no es la excepción y bajo las circunstancias del país al final del 2009, resulta verdaderamente trascendente iniciar con un proceso ordenado, sostenido y consistente de inversiones públicas y privadas, que contribuyan a llevar competitividad, oportunidades de desarrollo económico y social a todas las regiones del país". De lo anterior se puede deducir que el desarrollo de la aeronáutica civil es necesario para lograr la visión de país ya que la misma está implícitamente relacionada con los temas de transporte, infraestructura productiva, competitividad y desarrollo económico. Por lo tanto desde 2010, la oportunidad de desarrollar y fortalecer la aeronáutica civil nacional se encuentra presente y al igual como se menciona en la Visión de País (2010-2038) y el Plan de Nación (2010-2022), el desarrollo y fortalecimiento de la aeronáutica civil se debe de iniciar con un proceso ordenado, sostenido y consistente, y es aquí donde debe estar presente el Consejo Aeronáutico Nacional, como órgano consultivo que aglutina distintos sectores de la sociedad hondureña y pueda guiar el proceso para el desarrollo del sector aeronáutico en Honduras.

\section{¿Qué sucede con el CAN?}

En la actualidad, muchas de las personas que se desenvuelven día a día en la industria aeronáutica nacional desconocen la existencia, en Ley, del Consejo Aeronáutico Nacional, y esto fue evidenciado en la misma actividad académica mencionada anteriormente, donde se reunió personal representativo de la concesionaria, Dirección General de Aeronáutica Civil, escuelas de aviación, líneas aéreas entre otras, incluyendo algunos expertos en temas aeronáuticos.

Dicho desconocimiento se determinó relevante como tema de investigación debido a que se consideró que el CAN podría ser un órgano institucional clave para el desarrollo de la aeronáutica civil en el país, en vista de que éste sería el espacio donde convergerían los diferentes sectores involucrados en el tema aeronáutico como también de aquellos que se ven afectados por la industria y por tanto contribuyendo con diferentes puntos de vista en la discusión sobre temas aeronáuticos nacionales por lo que se pretendió determinar a través de un anteproyecto de investigación la situación actual del Consejo Aeronáutico Nacional en el marco institucional de la aeronáutica civil nacional. 


\section{¿Qué es lo que se sabe del CAN?}

En el año 2004 se publicó en el diario oficial de Honduras "La Gaceta" la Ley de Aeronáutica Civil y a través de ésta en su artículo 28 del capítulo III se estableció la creación del Consejo Aeronáutico Nacional (CAN), un órgano consultivo cuya función principal es la de identificar, concertar y proponer recomendaciones que apoyen y contribuyan al logro de los objetivos de la Ley de Aeronáutica Civil sin entrar en conflicto con las atribuciones y funciones de la Dirección General de Aeronáutica Civil. Adicional a esto algunas de las funciones descritas en Ley para el CAN incluyen: Conocer de los informes anuales y de operación de la DGAC y dar opinión sobre los mismos sin perjuicio de las disposiciones de la Organización de Aviación Civil Internacional (OACl) al respecto; Llevar a cabo acciones de control social con respecto a la gestión pública de la aeronáutica civil; Desarrollar actividades de información y divulgación sobre los asuntos de su competencia y otras acciones de concertación y propuesta en el marco de la aeronáutica civil.

Según la Ley de Aeronáutica Civil, son integrantes del CAN: el Secretario de Estado en el Despacho de Obras Públicas, Transporte y Vivienda (actualmente el Secretario en Infraestructura y Servicios Públicos), quien será el coordinador; el Secretario de Estado en el Despacho de Turismo (forma parte del gabinete económico); el Secretario de Estado en el Despacho de Seguridad; un representante de la Cámara de Turismo; un representante de la Cámara de Hoteleros; un representante de las cámaras de comercio de Honduras; el Director General de Aeronáutica Civil (actualmente Director de la Agencia Hondureña de Aeronáutica Civil), quien actuará como secretario con derecho a voz únicamente; y, el Secretario de Estado en el Despacho de Defensa Nacional.

En vista de todo lo anterior, el desarrollo de la aeronáutica civil nacional implica discutir temas relevantes para la sociedad en general como ser la seguridad, el medio ambiente, turismo y economía; por tanto es necesaria la participación activa de un órgano colegiado que integre a diferentes sectores de la institucionalidad y sociedad hondureña para apoyar a la Dirección General de Aeronáutica Civil en la toma de decisiones dentro de su competencia, su fortalecimiento institucional y en gran medida para la gestión de una verdadera política de Estado en materia de aeronáutica civil orientada hacia el desarrollo de la misma como eje transversal del desarrollo nacional. Ese órgano colegiado se considera debe ser el Consejo Aeronáutico Nacional (CAN). 


\section{Metodología Científica}

Para lograr definir la situación actual del Consejo Aeronáutico Nacional en el marco institucional de la aeronáutica civil nacional, la identificación de los factores que han impedido su funcionalidad y brindar recomendaciones en base a lo implementado en otros países, se llevó a cabo una investigación descriptiva a través de la cual se busca especificar propiedades, características y rasgos importantes de cualquier fenómeno que se analiza, en este caso particular, la situación actual del Consejo Aeronáutico Nacional incluyendo sus integrantes y marco normativo. Se utilizaron algunas técnicas de recolección de datos como la observación cualitativa, llevando a cabo una reflexión permanente, prestándose atención a los detalles, sucesos, eventos e interacciones, sobre todo en las discusiones dadas en el marco del Diplomado en Gestión de Sistemas Aeroportuarios. Se complementó con la recopilación de información relacionada con el CAN y disponible de forma pública, (Ley de Aeronáutica Civil, Leyes y Códigos en materia de aeronáutica civil de países como Chile, Ecuador y República Dominicana, información disponible en internet) para luego añadir entrevistas específicas a personal que integra algunas de las principales instituciones que forman y se considera deben formar parte del CAN, pretendiendo contestar preguntas como: ¿EI CAN funciona de manera efectiva en la actualidad?, ¿Qué factores influyen en la implementación efectiva 0 no efectiva del CAN?, las entidades definidas en la Ley de Aeronáutica Civil como parte del CAN ¿conocen a cabalidad sus responsabilidades?, ¿Las entidades incluidas en el CAN por la Ley de Aeronáutica Civil tienen interés en continuar siendo parte del CAN?, ¿Qué otras entidades podrían formar parte del CAN para mejorar su funcionalidad?, ¿Cómo se implementan órganos u organizaciones similares al CAN en otros países con mayor desarrollo aeronáutico civil?, ¿Qué temas debería analizar de manera inmediata el CAN?

Debido a que el tema de la aeronáutica civil pertenece tanto al ámbito nacional como internacional y en el siglo XXI con el fenómeno de la globalización, se consideró imprescindible conocer las experiencias que otros países han tenido en este tema con el fin de aprender, adaptar, mejorar y adoptar buenas prácticas que complementen los esfuerzos nacionales, por esta razón se incluyó el análisis de experiencias de tres países de la región Latinoamericana como Chile, Ecuador y República Dominicana en relación a órganos colegiados similares al CAN de Honduras. 


\section{Hallazgos}

Como se ha mencionado, en el ámbito de la aeronáutica civil participan diversos actores propios del ámbito y otros que tienen relación directa o indirectamente con él. En este sentido, se realizaron entrevistas a una diversidad de personas, las cuales forman parte respectivamente de la concesionaria de los cuatro aeropuertos internacionales de Honduras, la Dirección General de Aeronáutica Civil y representantes del sector turismo.

Mediante las entrevistas se encontró que existe un consenso y consciencia en cuanto a que el desarrollo de la aeronáutica nacional es de gran beneficio para el país sobre todo en materia económica y aporta en el desarrollo de otros rubros como por ejemplo el turismo.

Por otro lado, algo muy curioso es que indicaron que han leído la Ley Aeronáutica Civil pero a su vez manifestaron desconocer el CAN, lo que demuestra que no es suficiente con que se establezca mediante ley sino que debe ser implementado para que pueda realmente ser conocido y cumplir las funciones para las que fue creado. Correlativamente, manifestaron desconocer la cantidad de veces en que se han dado reuniones del CAN.

En relación a un integrante muy importante dentro del CAN, la Dirección General de Aeronáutica Civil, indicaron que conocen las funciones y responsabilidades de la DGAC y conociendo las mismas manifestaron que ésta no está cumpliendo en su totalidad con sus funciones al igual que con una de sus responsabilidades en relación al CAN, la cual es brindar apoyo presupuestario para el funcionamiento del consejo, pues el mismo personal de la DGAC entrevistado desconoce la asignación presupuestaria por ley destinada al CAN. De igual manera, la mayoría coincide en que se debe de supervisar a la DGAC -actualmente la Agencia Hondureña de Aeronáutica Civil- para asegurar el cumplimiento de sus objetivos, metas y funciones. Conforme a lo anterior, la mayoría de los entrevistados indicaron que esta institución sí podría necesitar algún tipo de asesoramiento y/o supervisión, una de las funciones principales que debería tener el CAN previa supervisión de organismos internacionales.

Basándonos en las entrevistas y en la información disponible en otros documentos se puede resumir que el CAN no ha tenido reuniones, por lo que no se han tratado temas relevantes relacionados con el rubro aeronáutico ni se han resuelto problemas específicos a través del mismo. Se puede indicar en base a lo anterior que el CAN no ha influido de ninguna forma en la toma de decisiones sobre 
la aeronáutica nacional. Según información obtenida a través de las entrevistas, no se definió un presupuesto para el desarrollo de reuniones e implementación del CAN. En relación a sí existe voluntad política actualmente para la implementación del CAN, hay diversidad de opiniones, algunos indican que sí, otros indican que no, por lo que no se puede concluir si efectivamente hay o no voluntad política. En relación a la pregunta de si hay interés por parte de la DGAC -Agencia Hondureña de Aeronáutica Civil- en la implementación del CAN, se puede concluir que no hubo interés en el pasado pero con el cambio en la Dirección es posible que este tema pueda ser considerado. Dentro de los beneficios que traería a la aeronáutica civil la implementación del CAN, según el personal entrevistado, se incluyen los siguientes: Voluntad política para hacer cambios, mejor calidad en el servicio aeronáutico, interés de otras líneas aéreas en traer más aviones a los aeropuertos del país, actualizaciones de las reglamentaciones y acuerdos aeronáuticos, personal mejor capacitado, mejor uso del presupuesto, supervisiones adecuadas de la concesión, apoyo para la DGAC. La mayoría de los entrevistados indican que, como representantes de sus instituciones, si estarían interesados en formar parte activamente del CAN. La mayoría de los entrevistados de la Dirección General de Aeronáutica Civil indican que desconocen los beneficios que traería para su institución la activación del CAN, mientras que otros entrevistados encontraron los siguientes beneficios: La concesionaria podría estar al tanto de todo lo que pasa en el rubro aeronáutico; se crearían nuevos contactos y conexiones claves; se crearía mayor afinidad con los otros entes involucrados; formación de mejores profesionales para trabajar en la aeronáutica civil; incremento de estándares de seguridad operacional; conformación de SSP y SMS como lo exige la regulación de la aviación civil internacional; parte de las inversiones que se harían serían devueltas a la concesionaria, se generarían más empleos porque se desarrollarían nuevas áreas para mejorar el servicio en mención. El total de los entrevistados indica que su institución no ha generado propuestas en apoyo al CAN, sin embargo éstas estarían dispuestas a designar personal específico para formar parte del CAN.

En relación a los temas que se consideran claves para incluir en la agenda a tratar por el CAN, los entrevistados mencionan los siguientes: La re-categorización del país; capacitación de personal técnico aeronáutico; manejo de presupuestos; programas de capacitaciones; mejoras al sistema aeroportuario; planificación de futuras propuestas de aviación civil; designación de presupuesto para actividades específicas de aviación civil; agenda sobre supervisiones realizadas; revisión de metas y objetivos estratégicos y operacionales; estudios aeronáuticos para el desarrollo de la aviación civil; generación de empleos en el ambiente aeronáutico; atención al cliente; mejora de instalaciones y equipo; mejor supervisión de pro- 
cedimientos aeronáuticos (plataforma, pistas, instalaciones); supervisión a líneas aéreas y a empresas concesionarias con más rigor; situación del personal y autonomía de la DGAC.

En relación a la consulta sobre qué entidades deberían formar parte del CAN, los entrevistados mencionaron las siguientes: Concesionaria, aerolíneas, Secretaría de Industria y Comercio, empresa privada, escuelas de aviación, Ministerio Público. Es importante mencionar que uno de los entrevistados manifestó que la Concesionaria no debería formar parte del CAN, basándose en que el CAN está considerado como ente supervisor de la DGAC y que en este caso la DGAC es quien supervisa a la Concesionaria, por lo que podría haber conflicto de intereses. De igual forma otro entrevistado sugiere la separación de los integrantes del CAN en dos categorías, en los que generan información y los que toman las decisiones, segmentándolo de la siguiente manera: los que generan información - DGAC y Concesionaria; y los que debaten los temas - DGAC, COCESNA, Secretaría de Turismo, Secretarias y entidades que se relacionan directamente con la aviación civil.

Por otra parte, es importante tomar en cuenta que Honduras se encuentra en una posición estratégica geopolíticamente en Latinoamérica, una región homogénea en muchos aspectos. Por lo que se consideró la experiencia que otros países han tenido con órganos colegiados, como el CAN, encontrando características relevantes que hacen que éstos órganos funcionen y brinden resultados en este interesante ámbito para cada uno de sus respectivos países.

Para la investigación se consideraron tres países latinoamericanos pues el marco institucional de cada uno de ellos en aeronáutica civil se encuentra plenamente identificada y estructurada mediante leyes, códigos y órganos, destacándose el hecho de que éstos no son consultivos -como lo es el CAN- sino más bien son los responsables de la dirección superior de la aviación civil en cada uno de sus países; hablamos de Chile, Ecuador y República Dominicana. En el cuadro comparativo se pueden conocer estas características por cada uno de los órganos similares al CAN en Chile, Ecuador y República Dominicana. 


\begin{tabular}{|c|c|c|c|c|c|c|c|}
\hline País & Nombre & Año de Creación & $\begin{array}{l}\text { Ley de } \\
\text { Creación }\end{array}$ & Dependencia & $\begin{array}{l}\text { Tipo de } \\
\text { Organismo }\end{array}$ & Función principal & Integrantes \\
\hline Ecuador & $\begin{array}{l}\text { Consejo Nacional de } \\
\text { Aviación Civil }\end{array}$ & $\begin{array}{l}1951 \text { con la Junta } \\
\text { Consultiva de } \\
\text { Aviación Civil }\end{array}$ & $\begin{array}{l}\text { Ley de } \\
\text { Aviación Civil }\end{array}$ & 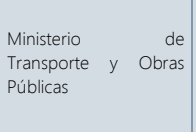 & $\begin{array}{l}\text { Organismo } \\
\text { ejecutivo }\end{array}$ & Emitir, regular y gestionar la política aeronáutica nacional & $\begin{array}{l}\text { - Presidencia de la República } \\
\text { - Ministro de Transporte y obras públicas } \\
\text { - Ministro de Turismo } \\
\text { - Ministro de Comercio Exterior } \\
\text { - Director General de Aviación Civil }\end{array}$ \\
\hline Chile & $\begin{array}{l}\text { Junta de Aeronáutica } \\
\text { Civil }\end{array}$ & $\begin{array}{l}\text { Se constituyó por } \\
\text { primera vez en } \\
1948 \text { y desde la } \\
\text { fecharra } \\
\text { sesionado en más } \\
\begin{array}{lr}\text { de r } & 1,800 \\
\text { ocasiones. }\end{array}\end{array}$ & $\begin{array}{l}\text { Ley Orgánica } \\
\text { de la Junta de } \\
\text { Aeronáutica } \\
\text { Civil de } 1960\end{array}$ & $\begin{array}{lr}\text { Ministerio } & \text { de } \\
\text { Transporte } & \text { y } \\
\text { Telecomunicaciones }\end{array}$ & $\begin{array}{l}\text { Órgano } \\
\text { administrativo } \\
\text { compuesto por } \\
\text { un Consejo y } \\
\text { una Secretaría } \\
\text { General }\end{array}$ & $\begin{array}{l}\text { Ejercer la dirección superior de la aviación civil, } \\
\text { gestionando políticas que promuevan su desarrollo y el } \\
\text { transporte aéreo comercial nacional e internacional } \\
\text { accesible, eficiente, competitivo, seguro y de calidad. }\end{array}$ & $\begin{array}{l}\text { - Ministro de Transporte y } \\
\text { Telecomunicaciones } \\
\text { - Director General de Aeronáutica Civil } \\
\text { - Sub-Secretario de Relaciones Exteriores } \\
\text { - Sub-Secretario de Desarrollo Social } \\
\text { - Director de Aeropuertos } \\
\text { - Presidencia de la República }\end{array}$ \\
\hline $\begin{array}{l}\text { República } \\
\text { Dominicana }\end{array}$ & Junta de Aviación Civil & $\begin{array}{lr}1969 & y \\
\text { ratificación en } \\
2006\end{array}$ & 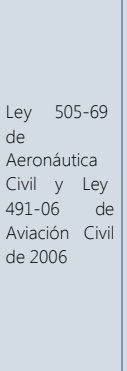 & $\begin{array}{l}\text { Poder Ejecutivo } \\
\text { (Presidencia, } \\
\text { Vicepresidencia y } \\
\text { Consejo de Ministros) }\end{array}$ & $\begin{array}{l}\text { Órgano asesor } \\
\text { y administrativo }\end{array}$ & $\begin{array}{l}\text { Establecer la política superior de la aviación civil en el país } \\
\text { al igual que regular los aspectos económicos del } \\
\text { transporte aéreo. }\end{array}$ & $\begin{array}{l}\text { - Presidente designado por el Poder } \\
\text { - Ejecutivo } \\
\text { - Secretario de Turismo } \\
\text { - Director General de IDAC } \\
\text { - Consultor jurídico del Poder Ejecutivo } \\
\text { - Especialistas en transporte aéreo } \\
\text { - Oficial General de la Fuerza Aérea } \\
\text { Dominicana } \\
\text { - Director General del Cuerpo } \\
\text { Especializado en Seguridad } \\
\text { - Aeroportuaria depresentante del Sector turístico } \\
\text { - Reprivado } \\
\text { - Director del Depto. Aeroportuario } \\
\text { - Secretario de Relaciones Exteriores. }\end{array}$ \\
\hline Honduras & $\begin{array}{l}\text { Consejo Aeronáutico } \\
\text { Nacional }\end{array}$ & $\begin{array}{l}\text { Establecido en el } \\
2004 \text { mediante el } \\
\text { art. } 28 \text { de la Ley } \\
\text { de Aeronáutica } \\
\text { Civil de Honduras }\end{array}$ & $\begin{array}{l}\text { Ley de } \\
\text { Aeronáutica } \\
\text { Civil de } \\
\text { Honduras }\end{array}$ & $\begin{array}{l}\text { Secretaría de Estado } \\
\text { de Infraestructura y } \\
\text { Servicios Públicos }\end{array}$ & $\begin{array}{l}\text { Órgano } \\
\text { consultivo }\end{array}$ & $\begin{array}{l}\text { Identificar y proponer al Poder Ejecutivo las } \\
\text { recomendaciones que contribuyan al logro de los } \\
\text { objetivos de la Ley de Aeronáutica Civil y realizar acciones } \\
\text { de control social sobre la gestión pública de la aeronáutica } \\
\text { civil. }\end{array}$ & $\begin{array}{l}\text { - Secretario de Infraestructura y Servicios } \\
\text { Públicos } \\
\text { - Secretario de Turismo } \\
\text { - Secretario de Seguridad } \\
\text { - Representante de la Cámara de Turismo } \\
\text { - Representante de la Cámara de } \\
\text { - Hoteleros } \\
\text { - Representante de las Cámaras de } \\
\text { Comercio e Industrias } \\
\text { - Director General de Aeronáutica Civil } \\
\text { - Secretario de Estado de Defensa } \\
\text { Nacional }\end{array}$ \\
\hline
\end{tabular}

\section{Cuadro comparativo 1: Estatuto de Autoridades Aeronáuticas seleccionadas}


En Chile, la Junta de Aeronáutica Civil es un órgano administrativo pluripersonal compuesto por dos entidades: el Consejo, como entidad resolutiva de composición interministerial y presidido por el Ministro de Transportes y Telecomunicaciones, y; la Secretaría General como entidad ejecutiva. Este órgano fue creado oficialmente en 1960, sin embargo se constituyó por primera vez el 29 de marzo de 1948 y desde entonces ha sesionado en más de 1,800 ocasiones. Como se mencionó en el párrafo anterior, la Junta de Aeronáutica Civil de Chile ejerce la dirección superior de la aviación civil a través de la gestión de políticas públicas que promueven su desarrollo y, especialmente, el del transporte aéreo comercial nacional e internacional. En la Figura 1 se puede ver el organigrama de la Junta Aeronáutica Civil de Chile.

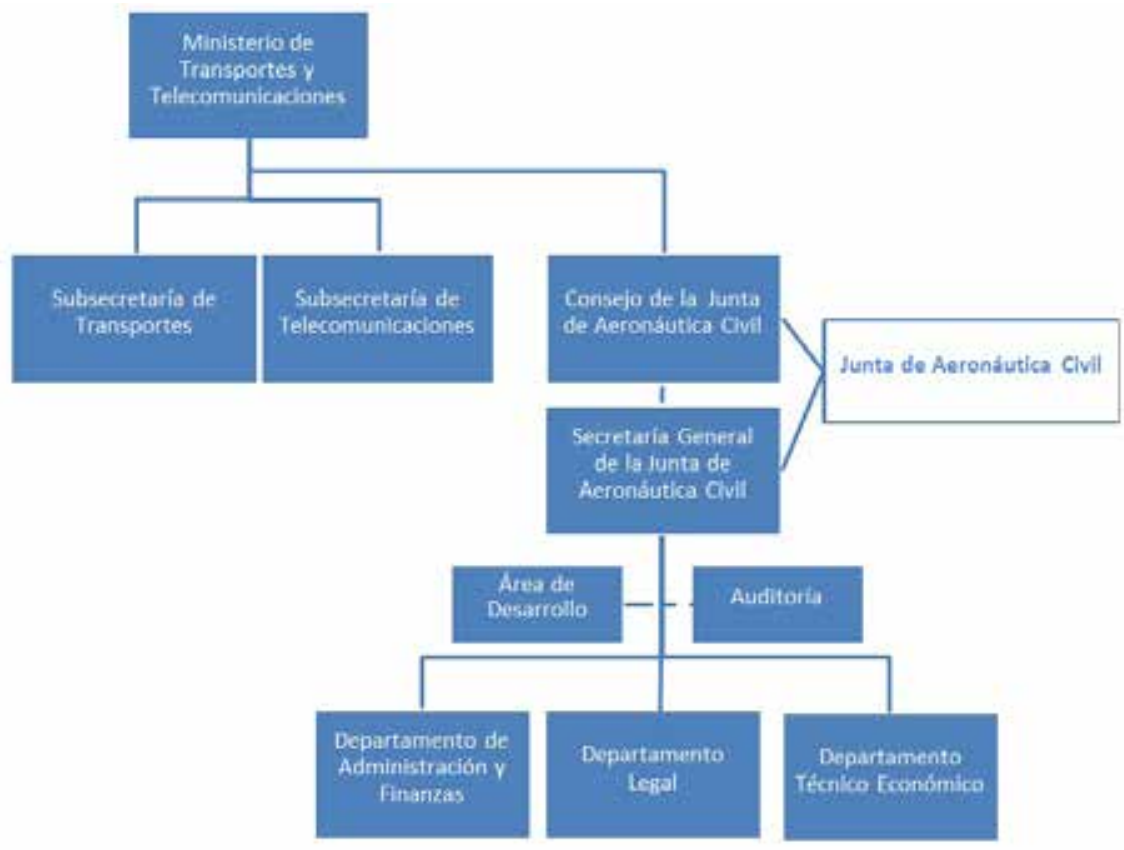

Figura 1. Organigrama de la Junta de Aeronáutica Civil de Chile

Dentro de los logros alcanzados por la Junta de Aeronáutica Civil se destacan la suscripción progresiva de acuerdos de servicios aéreos con el objeto de liberalizar los cielos chilenos, lo que ha generado un marco regulador estable y el cual ha permitido que los operadores aéreos puedan establecer planes de negocio en condiciones confiables, y han mantenido, desde el año 2012, acuerdos de servi- 
cios aéreos con más de 50 países alrededor del mundo. Estos acuerdos siguen en aumento.

En el caso de Ecuador, el Consejo Nacional de Aviación Civil (CNAC) es el organismo ejecutivo en materia aeronáutica mientras que la Dirección General de Aviación Civil es la entidad controladora y técnico-operativa. El CNAC fue creado en 1951, siendo en sus inicios la Junta Consultiva de Aviación

Civil. En el año 2000, el Congreso Nacional dictó varias reformas a la Ley de Aviación, entre las principales se encontraba la conversión del CNAC a un organismo regulador encargado de la política aeronáutica del país. En el 2006, se publicó la Ley Reformatoria a la Ley de Aviación Civil y Código Aeronáutico a través de la cual se llevó a cabo una nueva integración del CNAC, destacándose el hecho de que el Consejo sería presidido por el delegado del Presidente de la República. El contenido del primer artículo del Reglamento Interno del CNAC resalta pues declara que es el "organismo encargado de la planificación, regulación, control y de dictar las políticas de la aeronavegación comercial". En la Figura 2 se puede ver el organigrama del Consejo Nacional de Aviación Civil.

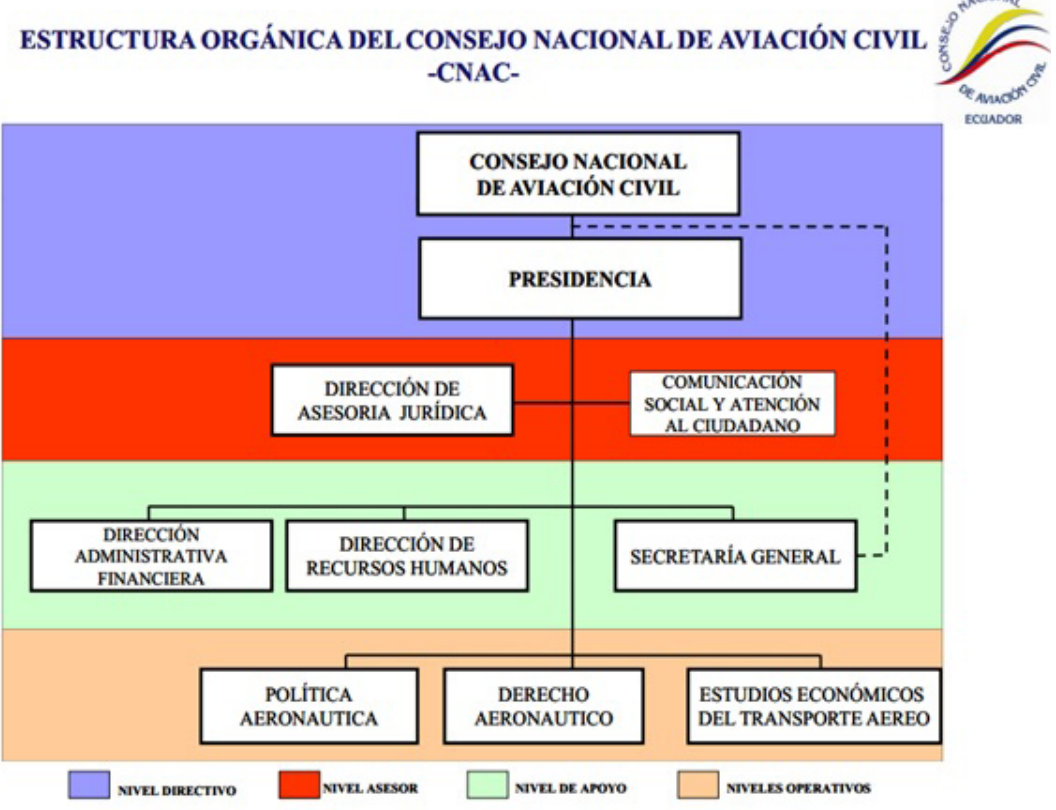

figura 2. Organigrama del CNAC 
Dentro de los logros más destacados del CNAC se puede mencionar la re-categorización del país en el año 2006 mediante un apoyo constante para la recertificación de las compañías aéreas ecuatorianas. En el 2006, la Administración Federal de Aviación de los Estados Unidos de América colocó a Ecuador en la categoría 1 luego de permanecer en la categoría 2 desde 1994. Desde el año 2007, el Consejo Nacional de Aviación Civil ha dirigido el Plan Nacional de Modernización de aeropuertos, sistemas de navegación y telecomunicaciones. Uno de los aspectos más relevantes dentro de este plan es que se determinó y finalizó la construcción del nuevo aeropuerto de Quito.

Por otra parte, en República Dominicana encontramos a la Junta de Aviación Civil (JAC). La JAC fue creada en 1969, como un organismo asesor del Poder Ejecutivo y que tenía a su cargo la política superior de la aviación civil en el país. En el año 2006, el Congreso Nacional la nueva Ley No. 491-06 de Aviación Civil con la cual se derogaba la ley anterior. Lo importante en relación a lo anterior es que con la nueva ley, la JAC tiene como responsabilidad principal establecer la política superior de la aviación civil en el país al igual que regular los aspectos económicos del transporte aéreo. Entre sus funciones se pueden mencionar las siguientes: Definir las políticas y estrategias para el desarrollo del transporte aéreo, y; Recomendar al Poder Ejecutivo la fijación de tasas y derechos aeronáuticos. De todos los órganos colegiados estudiados, este tiene el mayor número y diversidad de integrantes, siendo un total de 11 representantes de diferentes instituciones de distintos sectores. Ver Figura 3 para ver el organigrama de la JAC. 


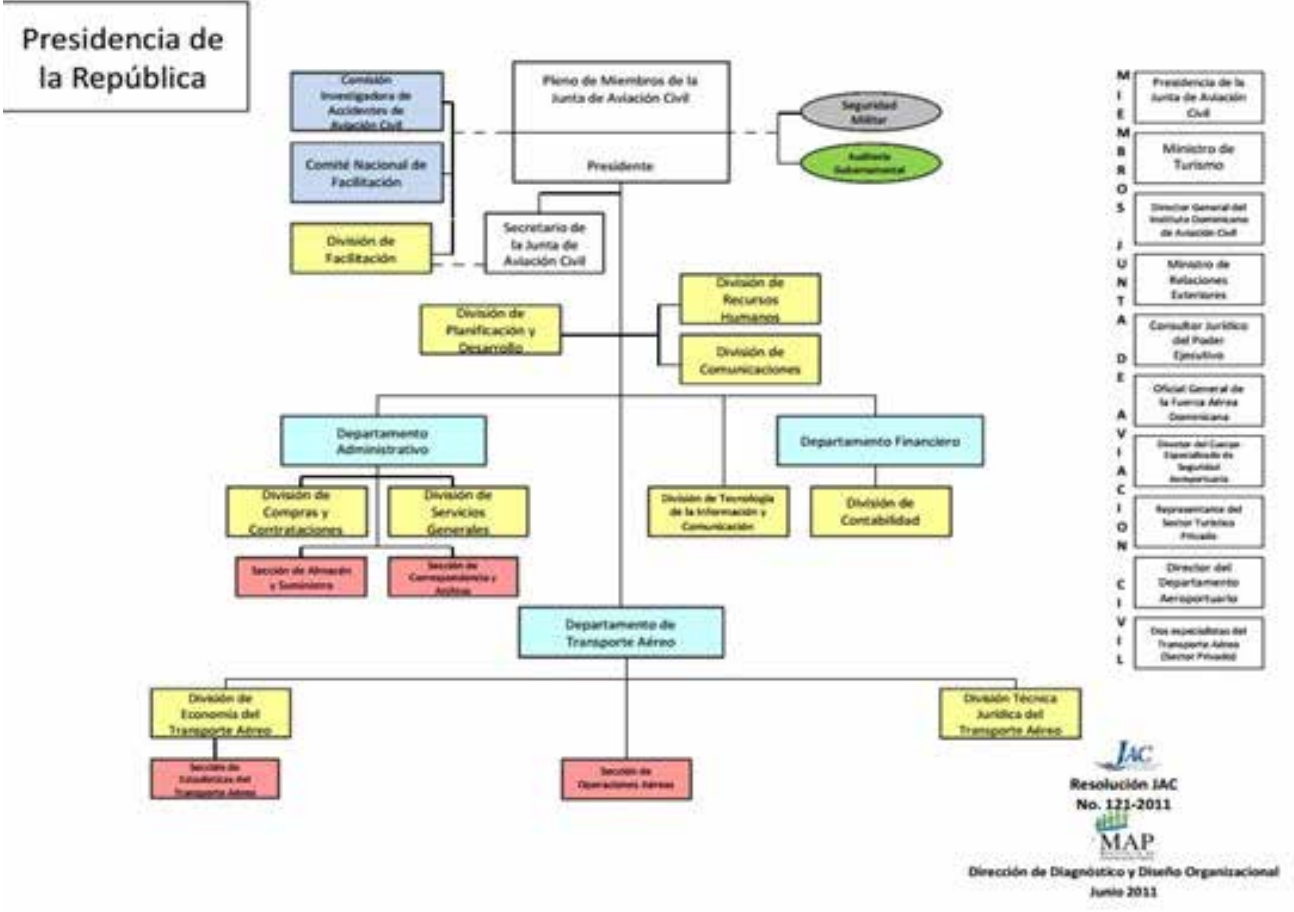

Figura 3 Organigrama de la JAC-República Dominicana

\section{Discusión}

A través del cuadro comparativo 1, se pueden observar arraigadas diferencias entre los consejos aeronáuticos de Ecuador, Chile y República Dominicana con el Consejo Aeronáutico Nacional de Honduras.

Una de ellas y que consideramos como la más importante es que los consejos aeronáuticos en los países antes mencionados, son órganos ejecutivos y administrativos, es decir que sus decisiones y políticas deben ser implementadas por todas aquellas instituciones involucradas en las mismas. En cambio, el CAN de Honduras es un órgano colegiado consultivo, contrario a un rol ejecutivo y/o administrativo, es decir que sus acuerdos y/o decisiones no son vinculantes. Relacionado a lo anterior, se encuentran las funciones que desempeña cada consejo aeronáutico en su respectivo país. 
En el caso de Chile, Ecuador y República Dominicana, los consejos aeronáuticos dictan y dirigen las políticas públicas de la aeronáutica civil mientras que la función principal del CAN en Honduras se limita a proponer recomendaciones y llevar a cabo acciones de control social en relación al tema aeronáutico civil.

Otra diferencia encontrada es la fecha de creación de los consejos aeronáuticos, pues en Chile, Ecuador y República Dominicana, estos organismos se crearon a mediados del siglo XX. En cambio, el CAN de Honduras se creó a principios del siglo XXI. Se puede decir que existe una abismal diferencia en la experiencia adquirida por Chile, Ecuador y República Dominicana a través de sus juntas y/o consejos aeronáuticos en relación al CAN, por ejemplo la Junta de Aeronáutica Civil en Chile ha sesionado en más de 1,800 ocasiones mientras que en la actualidad se desconoce cuántas veces lo ha hecho el CAN. Por lo tanto la sinergia creada a lo largo de los años por los consejos aeronáuticos de Ecuador, Chile y República Dominicana resulta beneficiosa para estos países, pues han desarrollado procedimientos que a su vez han permitido el funcionamiento de los consejos aeronáuticos, contrario al CAN cuyo reglamento no ha sido establecido aún por sus integrantes.

Con la Junta de Aviación Civil de República Dominicana se puede observar una significativa similitud ya que a través del cuadro comparativo se puede deducir que el sector turismo tiene una participación importante, sin embargo esto no es de extrañar pues ambos países dependen en gran medida del sector turístico.

Es importante destacar que el CAN aporta algo innovador en relación a los demás consejos aeronáuticos estudiados. El Consejo Aeronáutico Nacional de Honduras integra no sólo a instituciones gubernamentales sino que también representantes de la sociedad civil como los son las cámaras de comercio e industria y cámara de turismo. Sin duda alguna esto podría representar un aspecto importante para el mismo funcionamiento del CAN pues sus recomendaciones y decisiones estarían respaldadas por la misma sociedad civil, ya que ésta participa en la proposición de las mismas. En cambio, los consejos aeronáuticos de Ecuador y Chile no integran a organizaciones de sociedad civil por lo que es muy probable que sus políticas y decisiones deban ser ampliamente socializadas previo a su implementación o cumplimiento.

Al realizar el análisis de documentos nacionales se puede afirmar que existe la descripción del Consejo Aeronáutico Nacional en la legislación hondureña como se puede observar en la Ley de Aeronáutica Civil, donde se detalla quienes deben ser sus integrantes y cuáles son sus funciones. Sin embargo no existe ma- 
yor detalle sobre los procedimientos propios del consejo dado que no se encontró su reglamento interno, a través del cual se debería establecer las funciones de sus miembros y procedimientos. De igual forma, no se encontró documentación que indique que se asignó presupuesto para el funcionamiento del CAN constituido por Ley, ni documentos que indiquen que dicho Consejo Aeronáutico Nacional se reunión alguna vez, que temas trataron, que problemas solucionaron, etc.

Por otra parte los consejos/juntas similares al CAN han sido implementados por lo menos en países como Chile, Ecuador y República Dominicana, como entes ejecutivos, administrativos y de apoyo a sus direcciones de aeronáutica 0 entidades similares. En este sentido, el Ing. Samuel Véliz, experto chileno en el tema aeroportuario con años de experiencia, apoya la posición de que el carácter consultivo del Consejo de Aeronáutica Nacional de Honduras es una limitante y a la vez debilita su funcionamiento. Es por ello que él recomienda que este órgano, al igual que otros ya estudiados, dependan directamente de la Presidencia de la República con el objetivo de que sus decisiones tengan mayor peso al igual que se le brinde la debida atención desde las autoridades superiores del país. Por otro lado señaló que otra posible razón por la cual el CAN no ha sido implementado es que existen entidades del Estado que temen la discusión de ciertos temas por organizaciones externas y por tanto no desean la intromisión en sus asuntos pues les producen complicaciones legales, administrativas, etc.

Al revisar las estructuras de los consejos aeronáuticos o de aviación civil y las juntas de aeronáutica 0 aviación civil en otros países se puede afirmar que éstos son muy similares puesto que son órganos colegiados, es decir integrados por representantes de diferentes unidades/entidades de diferentes sectores y se ubican en un nivel superior. La diferencia entre estos tipos de organismos radica en sus atribuciones y dependencias.

Con base a la información disponible al público en general y las entrevistas realizadas se puede confirmar que el Consejo Aeronáutico Nacional no ha sido implementado.

Por otra parte, se puede apreciar un interés por parte de la Universidad Nacional Autónoma de Honduras, a través del Depto. de Ciencia Aeronáuticas de la Facultad de Ciencias Espaciales, en dar a conocer la necesidad de activar el CAN, esto se evidencia al presentar la investigación respecto al tema en el VIII Congreso de Investigación Científica de la UNAH, una de las actividades con las que se ha logrado dar a conocer cada vez más y a un mayor número de personas la necesidad real de que este órgano colegiado comience a cumplir las funciones 
para las que fue creado mediante ley desde hace una década. Poco a poco se está creando conciencia del efecto dominó que trae el desarrollo del rubro aeronáutico en otros sectores económicos del país; se está atrayendo la atención de personas que pueden ser elementos claves en el desarrollo aeronáutico del país para que tomen la decisión o hagan saber a quien corresponda que hay una forma eficiente de lograrlo y una forma casi inmediata es haciendo cumplir lo establecido en ley, activando el órgano que permita que todos las instituciones que afectan o se ven afectadas por el rubro aeronáutico expresen su opinión, dar a conocer sus metas individuales y mediante consenso tomar decisiones que conlleven al logro de metas colectivas, a través del desarrollo simultáneo 0 al menos el desarrollo vinculado de varias instituciones a través de un mismo producto o servicio aeronáutico.

Con todas las facilidades tecnológicas de hoy en día se puede conocer y adaptar lo hecho en otros países de manera que se pueda crear un modelo de consejo aeronáutico que pudiera llegar a ser incluso replicado a nivel regional 0 , ¿por qué no?, que este modelo sea el primer consejo regional aeronáutico. El cielo es el límite, claro, cuando no hablamos de aeronáutica.

De igual forma es necesario indicar que el CAN como fue concebido originalmente, posiblemente ya no cubra las necesidades actuales, por lo que analizar cómo se implementa en otros países es clave, para poder poner en funcionamiento un órgano consultivo adaptado a las necesidades reales del país y de la región incluso y que sea realmente eficiente.

Para llevar a cabo el "sueño" de desarrollar el rubro aeronáutico a través del Consejo Aeronáutico Nacional es necesario obtener el apoyo del Poder Ejecutivo, por lo que es labor permanente y clave hacer llegar la idea de activar el CAN a la institución definida como cabeza del mismo en Ley, la actual Secretaría de Infraestructura y Servicios Públicos (INSEP) para que una vez analizado por su parte se pueda obtener el impulso desde el gobierno para su activación.

\section{Conclusiones}

La investigación, en la cual se basa el presente artículo, concluye apoyándose en la información obtenida a través de los instrumentos de recopilación de datos (encuestas, entrevistas abiertas) documentos específicos (Ley de Aeronáutica Civil), e información en Internet que: La situación actual del Consejo Aeronáutico Nacional es que no ha sido implementado desde su creación en Ley, no funciona en la actualidad. 
Dentro de los factores que influyen en la implementación del CAN se encontró la falta de voluntad política en el pasado, la falta de interés en la implementación por parte de sus miembros, la falta de información sobre el CAN, la falta de socialización del tema de la aeronáutica civil, la inexistencia del reglamento interno y la falta de un presupuesto.

Respecto a países como Ecuador, Chile y República Dominicana funcionan órganos colegiados similares al Consejo Aeronáutico Nacional que a diferencia del CAN, éstos no tienen carácter consultivo sino más bien ejecutivo y administrativo, es decir, que sus acuerdos y decisiones son vinculantes y más importante aún son los encargados de plantear y dar seguimiento a las políticas y estrategias para el desarrollo de la aeronáutica civil en sus respectivos países. De esta manera, se puede concluir que este es un aspecto que limita en gran medida la funcionalidad del CAN, en vista de que teniendo la participación de representantes de diferentes sectores de la sociedad no aprovecha la misma para desarrollar políticas integrales en materia de aeronáutica civil dado que la Ley no lo faculta para tal efecto.

En este sentido y dado los cambios recientes en la estructura del Poder Ejecutivo en adición a las tendencias globales en la aviación civil internacional tales como seguridad y medio ambiente, se concluye que la integración del CAN no está acorde con las actuales necesidades del país en el ámbito aeronáutico civil, pues no tiene representación de importantes instituciones como la Secretaría de Recursos Naturales y Ambiente (SERNA), autoridad nacional en materia ambiental, entre otras.

\section{Propuestas}

Adicional a lo anterior en la investigación se recomienda, en base a las conclusiones planteadas, lo siguiente:

Realizar una investigación profunda, objetiva y precisa sobre la factibilidad de la implementación del Consejo Aeronáutico Nacional y su efecto en el desarrollo integral de la aeronáutica civil nacional a fin de determinar sí éste ocasionará alguna diferencia positiva en la realidad nacional respecto al ámbito aeronáutico civil.

A fin de contrarrestar los factores negativos que afectan la implementación del Consejo Aeronáutico Nacional se deben realizar acciones y actividades en promoción de la aeronáutica como tema de desarrollo de país dentro de las cuales se involucren no sólo a los miembros del CAN sino que también a las autoridades nacionales a nivel superior como el Presidente de la República, Presi- 
dente del Congreso Nacional, Secretario de Infraestructura y Servicios Públicos y el Director General de Aeronáutica Civil, teniendo como base la actividad académica desarrollada por la UNAH, siendo ésta la autoridad en educación superior del país que aglutina las diversas áreas del conocimiento y dado que es una institución que goza de la credibilidad y confianza de la sociedad hondureña.

Se deberá plantear por parte de algún integrante del Consejo Aeronáutico Nacional, siendo la más indicada la Agencia Hondureña de Aeronáutica Civil, una agenda a ejecutar en la cual se gestione reuniones de manera individual con los principales miembros del CAN a fin de socializar las responsabilidades que la Ley de Aeronáutica Civil les otorga al ser parte del CAN y señalando la importancia tanto para el país como para sus instituciones. Lo anterior, se espera, no tendrá mayor complicación o dificultad considerando que algunos miembros han manifestado estar interesados en el tema.

Sí la investigación recomendada en el primer numeral de esta sección concluye que la implementación del Consejo Aeronáutico Nacional es factible para el desarrollo integral de la aeronáutica civil nacional, se deberá, mediante reforma a la Ley de Aeronáutica Civil, elevar el carácter del CAN de un órgano consultivo a un órgano ejecutivo y cuyas funciones y/o responsabilidades se enmarquen dentro de este mismo carácter, teniendo como pilar fundamental la responsabilidad de plantear, definir y acordar una política de Estado para el desarrollo de la aeronáutica civil en el país en la cual sus miembros tengan tareas y roles claros en la ejecución de la misma.

De implementarse el Consejo Aeronáutico Nacional, sus primeras sesiones deberán tener como objetivo la introducción de sus miembros al ámbito aeronáutico civil, la creación del reglamente interno y el establecimiento de una agenda que refleje la priorización de los temas que el CAN debe discutir, como los que plantea la presente investigación.

Llevar a cabo una reforma a la Ley de Aeronáutica Civil a fin de incorporar nuevos miembros al Consejo Aeronáutico Nacional con el fin de fortalecer al mismo, pues deben tomar en cuenta a organizaciones y autoridades en temas que en la actualidad son tendencias globales y a la vez temas de interés nacional como el medio ambiente, desarrollo de servicios aéreos y seguridad. Asimismo se deberá actualizar a los miembros ya mencionados por la Ley debido al cambio significativo de la estructura del gobierno central, como el caso de la Secretaría de Turismo, la cual desaparece como Secretaría de Estado para formar parte del gabinete sectorial de desarrollo económico y sobre todo la conversión de la Dirección General 
de Aeronáutica Civil a la Agencia Hondureña de Aeronáutica Civil. Más importante aún es que el Consejo Aeronáutico Nacional debe incorporar las instituciones que forman parte del Sistema Aeronáutico Nacional como Interairports S.A. como concesionaria de los cuatro aeropuertos internacionales de Honduras, Asociación para el Desarrollo Aeronáutico y Estudio de Honduras (ADAEH), Corporación Centroamericana de Servicios de Navegación Aérea (COCESNA), Universidad Nacional Autónoma de Honduras (UNAH) y Universidad de Defensa de Honduras (UDH), aerolíneas y el Museo del Aire.

\section{Bibliografía}

- Schmelkes, C. y Elizondo Schmelkes, N. 2010. Manual para la presentación de anteproyectos e informes de investigación. Tercera edición. Oxford University Press México S.A. de CV. DF, México

- Fernández Collado, C., Baptista Lucio, P., Hernández Sampieri, R. 2010. Metodología de la Investigación. Quinta edición. McGraw-Hill/Interamericana Editores S.A. de C.V. DF, México.

- Ley No. 505-69. Ley de Aeronáutica Civil. República Dominicana. [Publicado en la Gaceta Oficial del 22 de noviembre de 1969].

- Ley No. 491-06. Ley de Aviación Civil. República Dominicana. [Publicado en la Gaceta Oficial del 28 de diciembre de 2006].

- Ley 18916. Aprueba Código Aeronáutico. Ministerio de Justicia. Santiago, Chile. [Publicado el 08 de febrero de 1990].

- Decreto con Fuerza de Ley No. 850. Fija el texto refundido, coordinado y sistematizado de la Ley No. 15.840, de 1964 y del Decreto con Fuerza de Ley No. 206 de 1960. Ministerio de Obras Públicas. Santiago, Chile. [Publicado el 25 de febrero de 1998].

- Decreto con Fuerza de Ley No. 241. Fusiona y reorganiza diversos servicios relacionados con la aviación civil. Ministerio de Hacienda. Santiago, Chile. [Publicado el 06 de abril de 1960].

- Decreto Legislativo No. 286-2009. Ley de Visión de País y Plan de Nación. Honduras. [Publicado en el diario oficial La Gaceta el 02 de febrero de 2010].

- Decreto Ejecutivo PCM-001-2014. Honduras. [Publicado en el diario oficial La Gaceta el 10 de marzo de 2014]. 
- Decreto Legislativo No. 55-2004. Ley de Aeronáutica Civil. Honduras. [Publicado en el diario oficial La Gaceta el 19 de mayo de 2004].

- Acuerdo No. 027. Reglamento Interno del Consejo Nacional de Aviación Civil. Consejo Nacional de Aviación Civil. Ecuador. 1992.

- Espacio Ecuador. Consejo Nacional de Aviación Civil de Ecuador. 2014. Página web. http://www.cnac.gob.ec/

- Junta de Aeronáutica Civil de Chile. 2014. Página web. http://www.jac-chile.cl

- Oficina Presidencial de Tecnologías de la Información y Comunicación (OPTIC). Junta de Aviación Civil de República Dominicana. 2014. Página web. http://www.jac.gob.do

- Dirección General de Aeronáutica Civil de Honduras. Asesoría Legal. 2014. Página web. http://www.dgachn.org/asesorialegal.html 